\title{
FLOW-INDUCED OSCILLATIONS OF TWO CYLINDERS IN TANDEM AND STAGGERED ARRANGEMENTS
}

\author{
S. MitTal ANd V. Kumar \\ Department of Aerospace Engineering, Indian Institute of Technology Kanpur \\ UP 208016, India
}

(Received 11 March 1999; and accepted 22 September 2000)

\begin{abstract}
A stabilized finite element formulation is employed to study flow-induced oscillations of a pair of equal-sized cylinders in tandem and staggered arrangement placed in uniform incompressible flow. Computations, restricted to 2-D, are carried out for Reynolds numbers 100 for various values of the structural frequency of the oscillator. The cylinders are separated by 5.5 times the cylinder diameter in the streamwise direction. For the staggered arrangement, the cross-flow spacing between the two cylinders is 0.7 times the cylinder diameter. In this arrangement, the downstream cylinder lies in the wake of the upstream one and therefore experiences an unsteady in-flow. Since the spacing between the two cylinders is beyond the critical value for proximity interference, it is expected that the upstream cylinder behaves like an isolated single cylinder, while the downstream one experiences wake-induced flutter. The $\operatorname{Re}=100$ flow leads to a very organized wake and large amplitude motion is observed for the downstream cylinder. The trajectory of the upstream cylinder resembles a figure-of-eight. The downstream cylinder shows a similar behaviour for the tandem arrangement. However, for the staggered arrangement, the trajectory of the rear cylinder resembles a tilted oval. Soft lock-in is observed in almost all the cases.

(C) 2001 Academic Press
\end{abstract}

\section{INTRODUCTION}

VORTEX-INDUCED OSCILLATIONS of bluff bodies has been investigated by various researchers in the last few decades. The interest in this class of problems emanates from its practical applications in a variety of engineering flows and also from the need to understand the complex phenomena exhibited by such systems. The flow past a stationary single cylinder, by itself, is associated with very rich vortex dynamics, and significantly different flow patterns are observed for various regimes of Reynolds numbers. The behaviour is further complicated if the cylinder is allowed to oscillate in response to the unsteady fluid forces acting on it.

The flow past an oscillating cylinder has been studied by various researchers in the past. Most of the investigations have been conducted using laboratory experiments. Some researchers have studied the flow past a cylinder subjected to forced oscillations, while others have looked at flow-induced vibrations. The interested reader is referred to the works by Toebes (1969), Griffin (1971), Tanida et al. (1973), Griffin \& Ramberg (1975), King (1977), Durgin et al. (1980), Chen (1987), Williamson \& Roshko (1988), Olinger \& Sreenivasan (1988), Ongoren \& Rockwell $(1988 a, b)$ and Blevins (1990). There have been fewer efforts using computational methods. Some of them are by Lecointe et al. (1987), Mittal et al. (1991), Mittal \& Tezduyar (1992), Mittal \& Kumar $(1998,1999)$, Chang \& Sa (1992) and Cetiner \& Unal (1995). A fairly comprehensive computational study for the vortex-induced oscillations of a single cylinder placed in a uniform flow has been reported in articles by Mittal \& Tezduyar (1992) and Mittal \& $\operatorname{Kumar}(1998,1999)$ for $\operatorname{Re}=325,1000,1500$ and 
$10^{4}$. These articles highlight the various phenomena associated with vortex-induced oscillations. Lock-in is observed for a range of structural frequencies of the oscillator; the vortexshedding frequency of an oscillating cylinder changes to the structural frequency as a result of the cylinder motion [see Koopmann (1967)]. Lock-in is also responsible for the phenomenon of hysteresis. The oscillation amplitude of a given oscillator, for a certain range of Reynolds number and close to the outer limits of the frequencies of lock-in, depends on whether the flow speed is decreased or increased during the experiment. Such phenomena have also been observed in laboratory experiments (Sarpkaya 1979), and either low- or high-amplitude oscillations may be realized for the same set of conditions depending on whether the flow is achieved by decreasing or increasing the flow speed.

The oscillations of the cylinder alter the flow significantly. In certain cases, the motion of the cylinder leads to modes of vortex shedding that are very different than the one usually observed for flow past a stationary cylinder. Sometimes, a competition between various modes of vortex shedding is observed. The choice of the mode(s) of vortex shedding is sensitive to the Reynolds number, oscillation amplitude and the ratio $F_{s} / F_{o} ; F_{o}$ is the vortex-shedding frequency for a stationary cylinder while $F_{s}$ is the structural frequency of the oscillator.

Another interesting feature observed for oscillators whose mass, compared to the mass of the surrounding fluid displaced by the cylinder is low, is soft lock-in (Mittal \& Kumar 1998, 1999). In lock-in, the vortex-shedding frequency of the oscillating cylinder changes from $F_{o}$ to $F_{s}$. However, in soft lock-in, the vortex-shedding frequency of the oscillating cylinder does not exactly match $F_{o}$; there is a slight detuning. This is one of the mechanisms of the nonlinear oscillator to self-limit its oscillation amplitude. It has been observed that the maximum oscillation amplitude of the spring-mass system does not change significantly even if the mass of the oscillator is reduced by a factor of 100 . The time-histories of the fluid mechanical forces acting on the oscillator get modified to self-limit the vibration amplitude. Some of the various mechanisms are a reduction in the time varying amplitude of the forces, introduction of an additional frequency component that acts like a fluid dynamic damping and the detuning between the structural and the vortex-shedding frequencies.

Fluid dynamic interaction between two stationary cylinders is quite sensitive to their relative arrangement (Zdravkovich 1977). If the cylinders are far apart, the flow around either of them is similar to that of an isolated cylinder. If the cylinders are close or if the second cylinder is adjacent to or within the wake of the first cylinder, the interference between the two can be one of the three types: proximity interference, wake interference, and proximity and wake interference. Proximity interference is usually seen for transverse arrangements and for closely spaced cylinders in staggered arrangements. In this type of interference, the formation of both vortex streets is affected by the two cylinders. Wake interference occurs for cylinders in tandem and staggered arrangements when the streamwise spacing between the two cylinders is sufficiently large. The upstream cylinder is unaffected by the presence of the downstream cylinder. The downstream cylinder lies in the wake of the upstream one and therefore suffers interference effects. When the downstream cylinder is close to the upstream cylinder and lies in its wake, both the cylinders affect the flow past each other. This results in proximity and wake interference and can occur for cylinders in tandem and staggered arrangements. The precise boundaries of different regimes are still not well known.

Some of the research that has gone into the understanding of flows past cylinders in various arrangements is reported in articles by Chen (1987), Zdravkovich (1977), Kim \& Durbin (1988), Tokunaga et al. (1992), Williamson (1985), Kiya et al. $(1980,1992)$ and Wu \& $\mathrm{Hu}$ (1993). Some of the interesting phenomena associated with such flows include coupled 
vortex streets, intermittent vortex shedding and biased flow that may lead to the flopping of the wake. Mittal et al. (1997) reported results for a systematic study involving cylinders in tandem and staggered arrangements with various spacings for Reynolds numbers of 100 and 1000. The wake is very organized at low Reynolds number and temporal periodicity is observed in all the cases that result in unsteady solutions. Two of the arrangements simulated by them exhibit wake interference. In one case, the cylinders are arranged in tandem with their centers separated by 5.5 times the cylinder diameter. In the second case, the spacing between the cylinders in the flow direction is the same as before, while the spacing in cross-flow direction is 0.7 times the cylinder diameter. In both cases, the upstream cylinder behaves very similarly to an isolated single cylinder, while the downstream one, that lies in the unsteady wake of the first one, experiences large unsteady forces. While the flow is temporally periodic in the near-wake of the upstream cylinder, the flow in the wake of the downstream cylinder does not exhibit such periodicity.

Flow-induced oscillations of two cylinders placed in a uniform flow is extremely complex and there have been very few systematic studies to understand such fluid-structure interactions. Most of the investigations, that these authors are familiar with, are based on laboratory experiments. Zdravkovich (1985) conducted a detailed investigation of flowinduced oscillations of two cylinders in various arrangements. The relative locations of the cylinders span all the three regimes for different types of interference. For each of the arrangement, the range of reduced velocity $\left(=1 / F_{s}\right)$ for which oscillations occur and the value for maximum amplitude were measured. The oscillation amplitudes of the two cylinders depend very strongly on their relative locations. The oscillations of one cylinder can affect the vortex shedding and synchronization (lock-in) of the other. It was observed that in some cases the displacement of the front cylinder is larger than the rear one, while in most of the cases the rear cylinder suffers larger amplitude oscillations. He classified the response of cylinders in three categories: (i) instability builds up rapidly to large amplitude primarily in streamwise direction, (ii) instability builds up slowly to a certain amplitude with oscillations mainly in streamwise direction, and (iii) instability grows gradually to large oscillation amplitudes primarily in cross-flow direction. This classification is related to the three regions of interference.

Jendrzejczyk et al. (1979) have conducted a detailed study with cylinders in tandem and transverse arrangement with spacing as 1.5 and 1.75 times the cylinder diameter. They observed four modes of vibration for the various cylinder arrangements, although different modes are dominant at different values of reduced flow velocity $\left(1 / F_{s}\right)$. They also observed that the fluid damping for the cross-flow vibrations vary with the vibration amplitude of the cylinders. King \& Johns (1976) reported a detailed study for two flexible cylinders in a tandem arrangement in water flow. The inter-cylinder spacing, in their investigation, varied from 1.5 to 7 times the cylinder diameter. In some of the experiments, the cylinders are coupled to each other via a horizontal member. It is rigid in certain cases and flexible in others. Three modes of vibration were observed for different ranges of $F_{s}$ : the fundamental mode in-line, the fundamental mode cross-flow, and the second normal mode in-line. The choice of modes is sensitive to the Reynolds number. For in-line motion of the cylinders, the Reynolds number must exceed 1200-1500. Tsui (1977) has proposed a mathematical model to simulate the wake-induced flutter of a cylinder. However, like several other models proposed by various researchers in the literature, the modelling of fluid dynamic forces is quite simple and does not incorporate the dependence on cylinder motion and other related parameters. Flow past a pair of stationary cylinders, itself, is not very well understood at this point and, therefore, it is the belief of these authors that it is almost impossible to construct a general model that predicts the response of the cylinders in any arbitrary arrangement. Some of the other works on flow-induced vibrations of two cylinders includes 
articles by Bokaian \& Geeola (1984a,b), Knisely \& Kawagoe (1990), Matsumoto et al. (1990) and Chen (1987).

In this article, we report our computational results for flow-induced oscillations of two cylinders placed in uniform flow at Reynolds number 100 in staggered and tandem arrangements. The cylinders are separated by 5.5 times their diameter in the streamwise direction. For the case of staggered arrangement, the cross-flow spacing between the two cylinders is 0.7 times the cylinder diameter. Both these arrangements correspond to the case of wake interference. The flow fields for the stationary cylinders in these arrangements were reported in an earlier article (Mittal et al. 1997).

The outline of the rest of the article is as follows. A brief review of the governing equations for incompressible fluid flow and for the motion of a rigid body under the influence of unsteady fluid forces is given in Section 2. Section 3 describes the stabilized finite element formulation of the governing equations. It is based on the space-time finite element method in which the finite element interpolation functions depend, both on space and time. This way the deformation of the spatial domain is taken into account automatically. This method, known as the deforming spatial domain/stabilized space-time (DSD/SST) technique, was introduced by Tezduyar et al. $(1992 a, b)$. Since then, it has been used for a variety of problems involving fluid-structure interactions and free-surfaces, for example, Mittal et al. (1991) Mittal \& Tezduyar (1994,1995), Mittal \& Kumar $(1998,1999)$.

To stabilize the computations against spurious numerical oscillations and to enable the use of equal-order-interpolation velocity-pressure elements the Galerkin/least-squares (GLS) stabilization technique is employed. Section 3 describes the finite element formulation incorporating these stabilizing terms. To accommodate the motion of the cylinders, and therefore the mesh, special mesh-moving schemes are used. Such special purpose mesh-moving strategies are very quick and allow one to completely do away with remeshing and the associated projection errors. The nonlinear equation systems resulting from the finite-element discretization of the flow equations are solved using the generalized minimal residual (GMRES) technique (Saad \& Schultz 1986) in conjunction with diagonal preconditioners. Results and discussions are presented in Section 4 and we end with some concluding remarks in Section 5.

\section{THE GOVERNING EQUATIONS}

Let $\Omega_{t} \subset \mathbb{R}^{n_{s i}}$ and $(0, T)$ be the spatial and temporal domains, respectively, where $n_{s d}$ is the number of space dimensions, and let $\Gamma_{t}$ denote the boundary of $\Omega_{t}$. The spatial and temporal coordinates are denoted by $\mathbf{x}$ and $t$. The Navier-Stokes equations governing incompressible fluid flow are

$$
\begin{aligned}
\rho\left(\frac{\partial \mathbf{u}}{\partial t}+\mathbf{u} \cdot \nabla \mathbf{u}-\mathbf{f}-\nabla \cdot \sigma\right. & =0 \text { on } \Omega_{t} \text { for }(0, T), \\
\nabla \cdot \mathbf{u} & =0 \text { on } \Omega_{t} \text { for }(0, T) .
\end{aligned}
$$

Here $\rho, \mathbf{u}, \mathbf{f}$ and $\boldsymbol{\sigma}$ are the density, velocity, body force and the stress tensor, respectively. The stress tensor is written as the sum of its isotropic and deviatoric parts:

$$
\boldsymbol{\sigma}=-p \mathbf{I}+\mathbf{T}, \quad \mathbf{T}=2 \mu \varepsilon(\mathbf{u}), \quad \varepsilon(\mathbf{u})=\frac{1}{2}\left((\nabla \mathbf{u})+(\nabla \mathbf{u})^{\mathbf{T}}\right),
$$

where $p$ and $\mu$ are the pressure and viscosity. Both the Dirichlet and Neumann-type boundary conditions are accounted for, represented as

$$
\mathbf{u}=\mathbf{g} \quad \text { on }\left(\Gamma_{t}\right)_{g}, \quad \mathbf{n} \cdot \boldsymbol{\sigma}=\mathbf{h} \quad \text { on }\left(\Gamma_{t}\right)_{h},
$$


where $\left(\Gamma_{t}\right)_{g}$ and $\left(\Gamma_{t}\right)_{h}$ are complementary subsets of the boundary $\Gamma_{t}$ and $\mathbf{n}$ is its unit normal vector. The initial condition on the velocity is specified on $\Omega_{t}$ at $t=0$ :

$$
\mathbf{u}(\mathbf{x}, 0)=\mathbf{u}_{0} \quad \text { on } \Omega_{0},
$$

where $\mathbf{u}_{0}$ is divergence free. A solid body immersed in the fluid experiences unsteady forces and in certain cases may exhibit rigid body motion. The motion of the body, in the two directions along the cartesian axes, is governed by the following equations:

$$
\begin{aligned}
& \ddot{X}+4 \pi F_{s} \zeta \dot{X}+\left(2 \pi F_{s}\right)^{2} X=\frac{C_{D}}{2 M} \text { for }(0, \mathrm{~T}), \\
& \ddot{Y}+4 \pi F_{s} \zeta \dot{Y}+\left(2 \pi F_{s}\right)^{2} Y=\frac{C_{L}}{2 M} \text { for }(0, \mathrm{~T}) .
\end{aligned}
$$

Here, $F_{s}$ is the reduced natural frequency of the oscillator, $\zeta$ is the structural damping coefficient, $M$ is the nondimensional mass of the body while $C_{L}$ and $C_{D}$ are the instantaneous lift and drag coefficients for the body, respectively. The free-stream flow is assumed to be along the $x$-axis. $\ddot{X}, \dot{X}$ and $X$ denote the normalized in-line acceleration, velocity and displacement of the body, respectively, while $\ddot{Y}, \dot{Y}$ and $Y$ represent the same quantities associated with the cross-flow motion. In the present study, in which the rigid body is a circular cylinder, the displacement and velocity are normalized by the diameter of the cylinder and the free-stream speed, respectively. The reduced natural frequency of the system, $F_{s}$ is defined as $2 f_{s} a / U_{\infty}$, where $f_{s}$ is the actual frequency of the oscillator, $a$ is the radius of the cylinder and $U_{\infty}$ is the free-stream speed of the flow. The nondimensional mass of the cylinder is defined as $M=m_{b} /\left(\rho_{\infty} a^{2}\right)$, where $m_{b}$ is the actual mass of the oscillator per unit length and $\rho_{\infty}$ is the density of the fluid. It may also be expressed as $M=\pi \rho_{s} / \rho_{\infty}$, where $\rho_{s}$ is the effective density of the material of the cylinder. The force coefficients are computed by carrying an integration, that involves the pressure and viscous stresses, around the circumference of the cylinder:

$$
\begin{aligned}
& C_{D}=\frac{1}{\frac{1}{2} \rho_{\infty} U_{\infty}^{2} 2 a} \int_{\Gamma_{\text {en }}}(\boldsymbol{\sigma n}) \cdot \mathbf{n}_{x} \mathrm{~d} \Gamma, \\
& C_{D}=\frac{1}{\frac{1}{2} \rho_{\infty} U_{\infty}^{2} 2 a} \int_{\Gamma_{\text {en }}}(\boldsymbol{\sigma n}) \cdot \mathbf{n}_{y} \mathrm{~d} \Gamma,
\end{aligned}
$$

Here, $\mathbf{n}_{x}$ and $\mathbf{n}_{y}$ are the Cartesian components of the unit vector $\mathbf{n}$ that is normal to the cylinder boundary $\Gamma_{\text {cyl }}$. These coefficients include the fluid dynamic damping and the added mass effect. The initial conditions for the displacement and velocity of the cylinder are specified at $t=0$ :

$$
\begin{array}{ll}
X(0)=X_{n} & \dot{X}(0)=\dot{X}_{0}, \\
Y(0)=Y_{0}, & \dot{Y}(0)=\dot{Y}_{0} .
\end{array}
$$

The equations governing the fluid flow are solved in conjunction with those for the motion of each of the two cylinders. The force acting on the body is calculated by integrating the flow variables on the body surface. The resulting drag and lift coefficients are used to compute the displacement and velocity of the body which are then used to update the location of the body and the no-slip boundary condition for the velocity field on the body surface. 


\section{FINITE ELEMENT FORMULATION}

To accommodate the motion of the cylinder and the deformation of the mesh, the stabilized finite element formulation is employed. In order to construct the finite element function spaces for the space-time method, we split the time interval $(0, T)$ into subintervals $I_{n}=\left(t_{n}, t_{n+1}\right)$, where $t_{n}$ and $t_{n+1}$ belong to an ordered series of. time levels: $0=t_{0}<t_{1}<\cdots<t_{N}=T$. Let $\Omega_{n}=\Omega_{t_{n}}$ and $\Gamma_{n}=\Gamma_{t .}$. We define the space-time slab $Q_{n}$ as the domain enclosed by the surfaces $\Omega_{n}, \Omega_{n+1}$, and $P_{n}$, where $P_{n}$ is the surface described by the boundary $\Gamma_{t}$ as $t$ traverses $I_{n}$. As is the case with $\Gamma_{t}$, the surface $P_{n}$ is decomposed into $\left(P_{n}\right)_{g}$ and $\left(P_{n}\right)_{h}$ with respect to the type of boundary condition (Dirichlet or Neumann) being imposed. For each space-time slab we define the corresponding finite element function spaces: $\left(\mathscr{S}_{\mathrm{u}}^{h}\right)_{n},\left(\mathscr{V}_{\mathrm{u}}^{h}\right)_{n},\left(\mathscr{S}_{p}^{h}\right)_{n}$, and $\left(\mathscr{V}_{p}^{h}\right)_{n}$. Over the element domain, this space is formed by using first-order polynomials in space and time. Globally, the interpolation functions are continuous in space but discontinuous in time.

The stabilized space-time formulation for deforming domains is then written as follows: given $\left(\mathbf{u}^{h}\right)_{n}$, find $\mathbf{u}^{h} \in\left(S_{\mathbf{u}}^{h}\right)_{n}$ and $p^{h} \in\left(\mathscr{S}_{p}^{h}\right)_{n}$ such that $\forall \mathbf{w}^{h} \in\left(\mathscr{V}_{u}^{h}\right)_{n}, q^{h} \in\left(\mathscr{V}_{p}^{h}\right)_{n}$,

$$
\begin{aligned}
\int_{Q_{n}} \mathbf{w}^{h} \cdot \rho\left(\frac{\partial \mathbf{u}^{h}}{\partial t}+\mathbf{u}^{h} \cdot \nabla \mathbf{u}^{h}-\mathbf{f}\right) \mathrm{d} Q & +\int_{Q_{n}} \boldsymbol{\varepsilon}\left(\mathbf{w}^{h}\right): \boldsymbol{\sigma}\left(p^{h}, \mathbf{u}^{h}\right) \mathrm{d} Q+\int_{Q_{n}} q^{h} \nabla \cdot \mathbf{u}^{h} \mathrm{~d} Q \\
+ & \sum_{e=1}^{n_{n}} \int_{Q_{n}} \frac{1}{\rho} \tau\left[\rho\left(\frac{\partial \mathbf{w}^{h}}{\partial t}+\mathbf{u}^{h} \cdot \nabla \mathbf{w}^{h}\right)-\nabla \cdot \sigma\left(q^{h}, \mathbf{w}^{h}\right)\right] . \\
& {\left[\rho\left(\frac{\partial \mathbf{u}^{h}}{\partial t}+\mathbf{u}^{h} \cdot \nabla \mathbf{u}^{h}-\mathbf{f}\right)-\nabla \cdot \sigma\left(p^{h}, \mathbf{u}^{h}\right)\right] \mathrm{d} Q } \\
+ & \sum_{e=1}^{n_{n}} \int_{Q_{n}^{k}} \delta \nabla \cdot \mathbf{w}^{h} \rho \nabla \cdot \mathbf{u}^{h} \mathrm{~d} Q+\int_{\Omega_{n}}\left(\mathbf{w}^{h}\right)_{n}^{+} . \\
& \rho\left(\left(\mathbf{u}^{h}\right)_{n}^{+}-\left(\mathbf{u}^{h}\right)_{n}^{-}\right) \mathrm{d} \Omega=\int_{\left(P_{n}\right) h} \mathbf{w}^{h} \cdot \mathbf{h}^{h} \mathrm{~d} P .
\end{aligned}
$$

This process is applied sequentially to all the space-time slabs $Q_{0}, Q_{1}, \ldots, Q_{N-1}$. In the variational formulation given by equation (12), the following notation is being used:

$$
\begin{gathered}
\left(\mathbf{u}^{h}\right)_{n}^{ \pm}=\lim _{\varepsilon \rightarrow 0} \mathbf{u}\left(t_{n} \pm \varepsilon\right), \\
\int_{Q_{n}}(\ldots) \mathrm{d} Q=\int_{I_{n}} \int_{\Omega_{\Omega_{n}}}(\ldots) \mathrm{d} \Omega \mathrm{d} t, \\
\int_{P_{n}}(\ldots) \mathrm{d} P=\int_{I_{n}} \int_{\Gamma_{n}}(\ldots) \mathrm{d} \Gamma \mathrm{d} t .
\end{gathered}
$$

The computations start with

$$
\left(\mathbf{u}^{h}\right)_{0}^{-}=\mathbf{u}_{0},
$$

where $\mathbf{u}_{0}$ is divergence-free.

In the variational formulation given by equation (12), the first three terms and the right-hand side constitute the Galerkin formulation of the problem. The series of elementlevel integrals involving the coefficients $\tau$ and $\delta$ are the least-squares terms that are added to the basic Galerkin formulation to ensure the stability of the computations. This type of stabilization is referred to as the Galerkin/least-squares (GLS) procedure and is a generalization of the Streamline-upwind/Petrov-Galerkin (SUPG) and pressure-stabilizing/ 
Petrov-Galerkin (PSPG) methods (Tezduyar et al. 1992). In the current formulation, $\tau$ and $\delta$ are given as

$$
\begin{gathered}
\left.\left.\tau=\frac{\left(2\left\|\mathbf{u}^{k}\right\|\right.}{h}\right)^{2}+\left(\frac{4 v}{h^{2}}\right)^{2}\right)^{-1 / 2} \\
\delta=\frac{h}{2}\left\|\mathbf{u}^{h}\right\| z,
\end{gathered}
$$

where

$$
=\begin{array}{ll}
\left(\frac{1}{3} \mathrm{Re}_{u}\right) & \mathrm{Re}_{u} \leq 3 \\
\mathrm{Re}_{u}>3
\end{array},
$$

and $\mathrm{Re}_{u}$ is the cell Reynolds number. Both stabilization terms are weighted residuals, and therefore maintain the consistency of the formulation. The sixth term in equation (12) enforces weak continuity of the velocity field across the space-time slabs.

The equations of motion for the oscillator given by (6)-(11) are also cast in the space-time formulation in the same manner as described in the work by Tezduyar et al. (1992) and Mittal (1992).

\section{RESULTS AND DISCUSSION}

All the computations reported in this article are carried out on the Digital work-stations at IIT Kanpur in 64 bit precision. Equal-in-order basis functions for velocity and pressure, that are bilinear in space and linear-in-time, are used and $2 \times 2 \times 2$ Gaussian quadrature is employed for numerical integration. The nonlinear equation systems resulting from the finite-element discretization of the flow equations are solved using the generalized minimal residual (GMRES) technique (Saad \& Schultz 1986) in conjunction with diagonal preconditioners.

The two equal-sized cylinders, of unit radii, reside in a rectangular computational domain whose upstream and downstream boundaries are located at 5 and 30 cylinder diameters, respectively, from the centre of the upstream cylinder. The upper and lower boundaries are placed at 10 diameters, each, from the centre of the first cylinder. The no-slip condition is satisfied for the velocity on the cylinder wall and free-stream values are assigned for the velocity at the upstream boundary. At the downstream boundary, we specify a Neumanntype boundary condition for the velocity that corresponds to zero viscous stress vector. On the upper and lower boundaries, the component of velocity normal to and the component of stress vector along these boundaries is the prescribed zero value. Computations are carried out for two different relative locations of the cylinders and Reynolds number 100. Reynolds number is based on the diameter of the cylinders, free-stream velocity and the viscosity of the fluid. The nondimensional distance between the centres of the two cylinders is denoted by $P / D$ in the flow direction and by $T / D$ in the cross-flow direction as shown in Figure 1 . In the rest of the article we will refer to the upstream cylinder as cylinder 1 and to the downstream one as cylinder 2 . The quantities with suffix 1 are associated with cylinder 1 , while those with suffix 2 are for cylinder 2 . Computations are carried out for two sets of cylinder arrangements. In one case, the two cylinders are arranged in tandem with $T / D=0$ and $P / D=5 \cdot 5$, while the other case is that of staggered arrangement with $T / D=0.7$ and $P / D=5.5$. The results for flow past stationary cylinders in these arrangements have been reported in an earlier article (Mittal et al. 1997). Here, results are presented for the vortex-induced oscillations of the two cylinders for various values of the structural 
$\boldsymbol{U}$
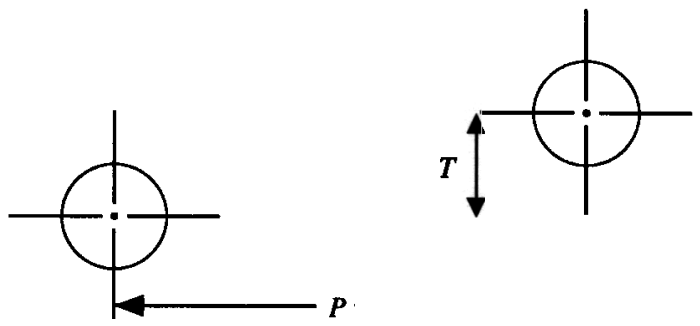

Figure 1. Description of the relative location of the two cylinders.

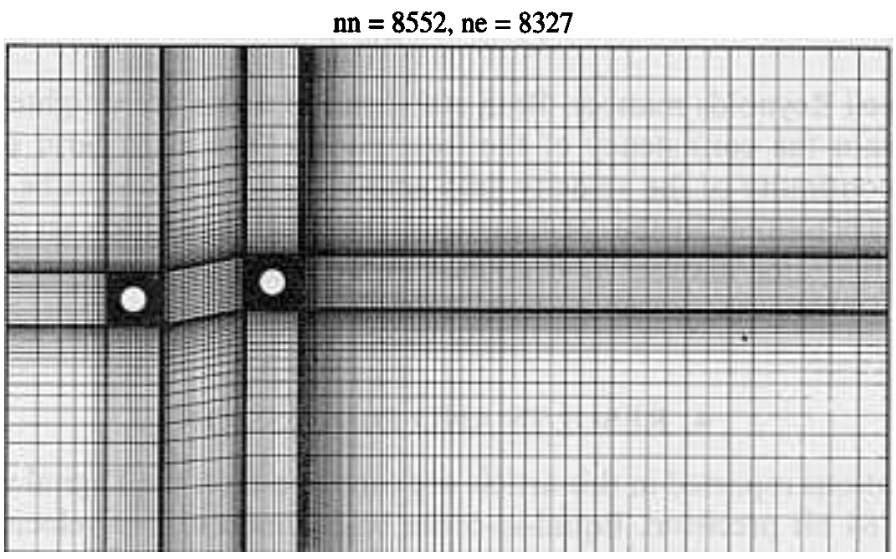

$\mathrm{nn}=8552, \mathrm{ne}=8327$

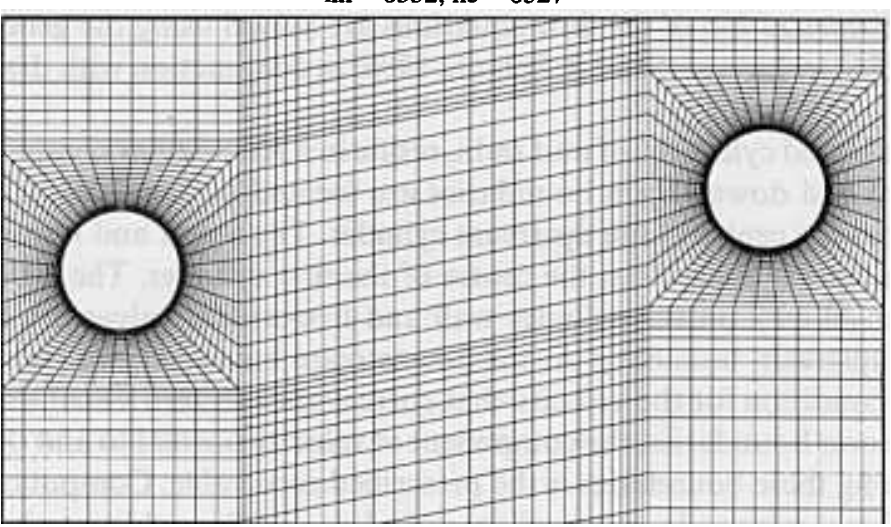

Figure 2. Flow past two cylinders in staggered arrangement, $T / D=0 \cdot 7, P / D=5 \cdot 5$ : finite element mesh with 8552 nodes and 8327 elements.

frequency of the spring-mass oscillator $\left(F_{s}\right)$ that are either equal or close to the vortexshedding frequency for the stationary cylinders $\left(F_{o}\right)$.

The flow calculations are carried out on a finite element mesh with 8552 nodes and 8327 quadrilateral elements. A picture of the mesh and its close-up is shown in Figure 2. Later, in the article, results are also presented for a more refined mesh to establish convergence of the computed results. The mesh has been designed to accommodate a mesh-moving scheme that eliminates the need for re-meshing. As can be observed from Figure 2, each of the two 
cylinders resides in a square box. The mesh within these boxes moves as a rigid body along with the cylinders. The external boundary of the domain is fixed and the motion of the cylinders is accounted for by relocating the nodes outside the square boxes. Therefore, the mesh close to the cylinders does not undergo any deformation and contributes to the accuracy of the resulting solution by preserving the gradients in the boundary/shear layers close to the solid body. It should be noted that with this mesh-moving scheme the connectivity of the mesh remains unaltered throughout the computations, thereby, eliminating the need for complex data structure(s).

The initial condition for all the computations is the fully developed unsteady solution past stationary cylinders in the same arrangement and at the same Reynolds number. The initial displacement and velocity of the two cylinders, in cross-flow and in-line directions, is assumed to be zero. At higher Reynolds numbers many of these flows are expected to have three-dimensional effects (Williamson 1996). For example, it was reported in Mittal et al. (1997) that compared to experimental results and those from 3-D calculations, 2-D computations for $\operatorname{Re}=1000$ that flow past a stationary cylinder overpredict the mean drag coefficient and the Strouhal number related to the vortex-shedding frequency. The non dimensional mass of each of the two cylinders is $M=4.7273$ and the structural damping coefficient is $\zeta=3.3 \times 10^{-4}$. These parameter values are same as those used for the studies involving single cylinder by Mittal \& Kumar $(1998,1999)$.

\subsection{Flow Past Two Cylinders in Tandem Arrangement; $T / D=0, P / D=5 \cdot 5$}

The solution for flow past stationary cylinders in this arrangement was reported in an earlier article (Mittal et al. 1997). The wake is quite organized and temporal periodicity is observed in the fully developed unsteady solution. The vortex shedding from the two cylinders is almost antiphase, i.e., as the first cylinder sheds a vortex from the upper surface, the second one sheds a counter-rotating one from the lower surface. The vortex-shedding frequency for the stationary cylinders $\left(F_{o}\right)$ is 0.168 . This value is quite close to that for a single cylinder at the same Reynolds number. Figure 3 shows the time histories of the lift and drag coefficients and the response of the cylinders for $F_{s}=0.168\left(=F_{o}\right)$ for the fully developed solution. The response of the upstream cylinder is very similar to that of a single cylinder as reported by Mittal \& Kumar (1998). The trajectory of the cylinder corresponds to a Lissajous figure-of-eight. The approximate values of the amplitude of the cross-flow and in-line oscillations are, respectively, 0.6 and 0.04 times the cylinder diameter. Compared to the stationary cylinder, the oscillating cylinder exhibits larger values of the mean and amplitude of the time-varying component of the drag coefficient and a smaller amplitude of the lift coefficient. The downstream cylinder lies in the unsteady wake of the upstream cylinder and experiences a galloping/flutter type of instability. The amplitudes of the cylinder oscillations are larger for the downstream cylinder. Beats can be observed in the time-histories of the lift and drag coefficients and response of the cylinder.

It is interesting to note that the nondimensional frequency associated with the variation of the lift coefficients and the cross-flow oscillations of both the cylinder is $0 \cdot 157$. This detuning between the structural frequency and the vortex-shedding frequency has been observed for single cylinders as well (Mittal \& Kumar 1999; Cetiner \& Unal 1995) and is one of the mechanisms of the oscillator to self-limit its oscillation amplitude. Mittal \& Kumar (1999) have referred to this phenomenon as soft lock-in. The detuning between the structural and vortex-shedding frequency reduces if the oscillator mass is increased. Figure 4 shows the pressure and vorticity fields at two time instants that correspond to the peak value of the cross-flow displacement of the downstream cylinder. The lower row of figures are for large values, while the upper row corresponds to the smaller values of the 

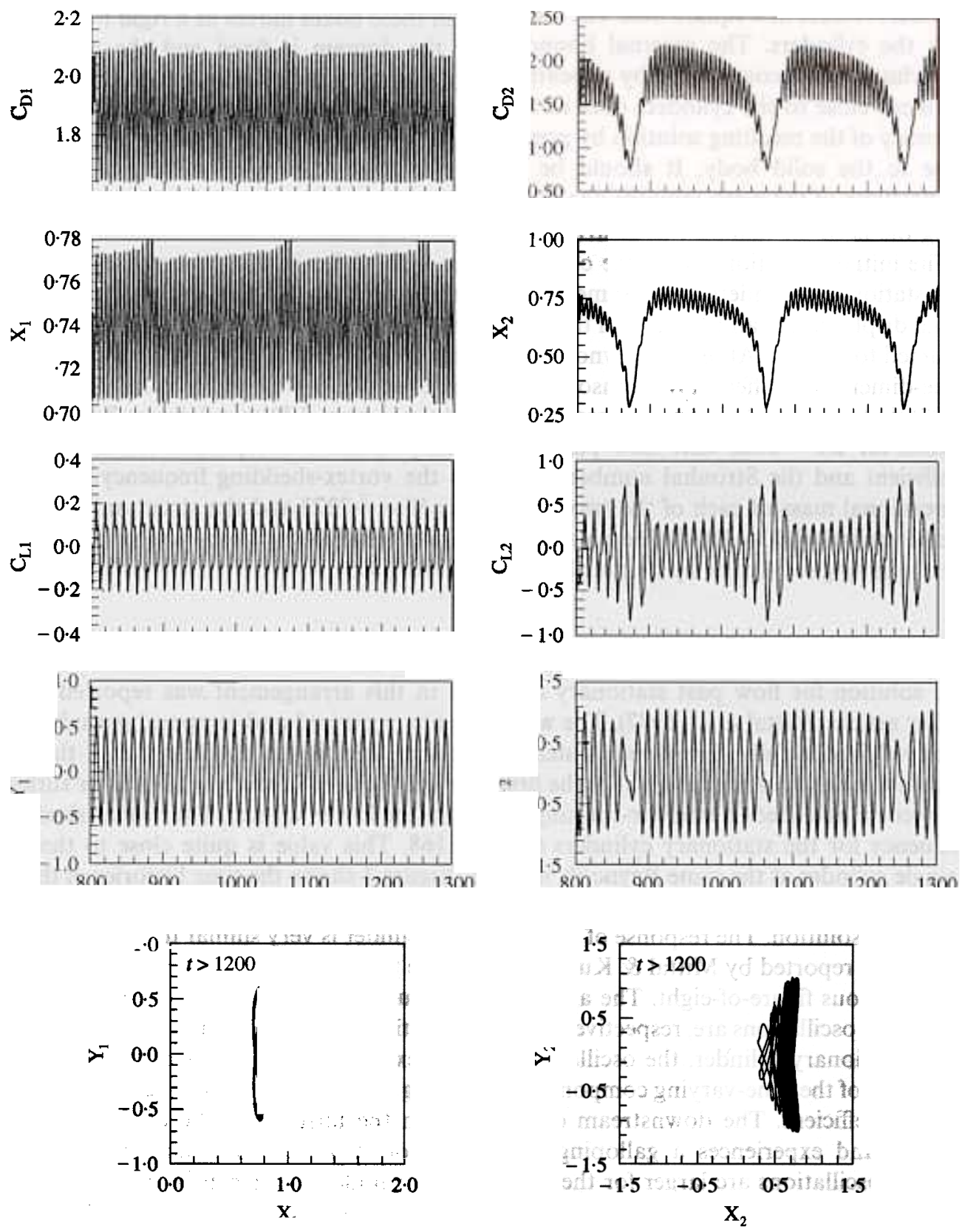

Figure 3. $\operatorname{Re}=100$ flow past two oscillating cylinders with $F_{s}=0 \cdot 168\left(=F_{o}\right)$ in tandem arrangement, $T / D=0, P / D=5 \cdot 5$ : time-histories of the drag and lift coefficients and the response of the cylinders.

amplitude of oscillations. The two sets of flow pictures are significantly different from each other. In the case of high-amplitude oscillations, a counter-clockwise rotating vortex released from the lower surface of the upstream cylinder is located below the lower surface of the downstream cylinder while it is at its peak location. This results in a low-pressure region on the lower surface of the downstream cylinder and causes it to exhibit large 

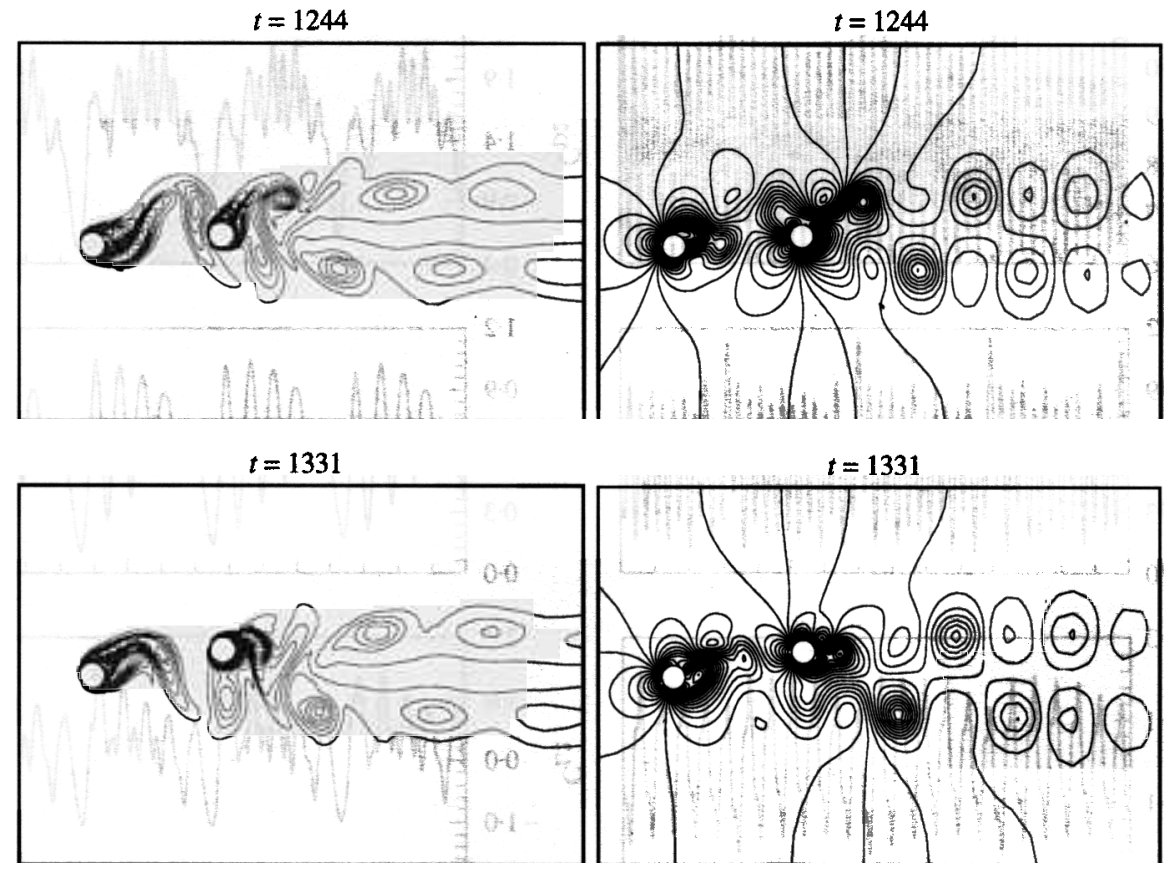

Figure 4. $\operatorname{Re}=100$ flow past two oscillating cylinders with $F_{s}=0.168\left(=F_{o}\right)$ in tandem arrangement, $T / D=0, P / D=5 \cdot 5$ : vorticity (left) and pressure (right) fields at the peak value of the cross-flow displacement of the downstream cylinder. The top row is for small, while the bottom row is for large amplitude oscillations.
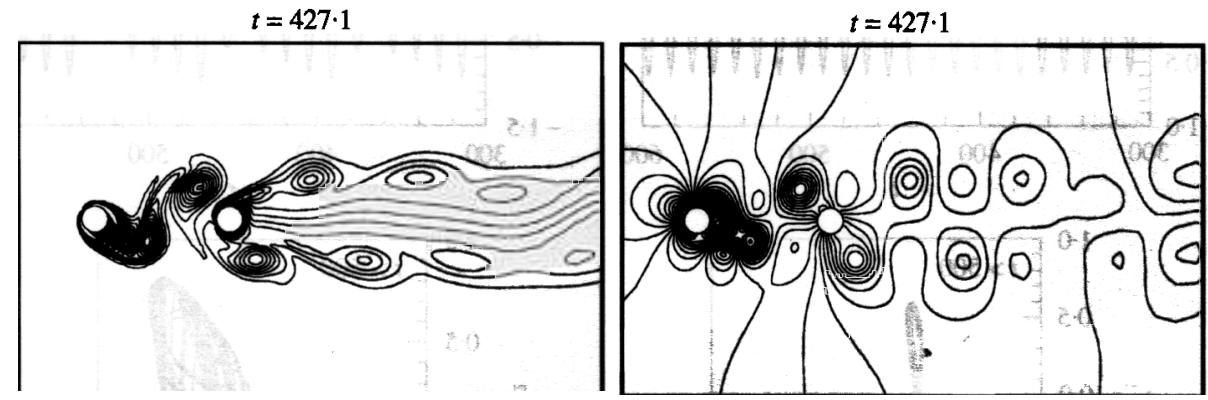

Figure 5. $\operatorname{Re}=100$ flow past two oscillating cylinders with $F_{s}=0.234\left(=1.4 F_{o}\right)$ in tandem arrangement, $T / D=0, P / D=5 \cdot 5$ : vorticity (left) and pressure (right) fields at the peak value of the cross-flow displacement of the upstream cylinder.

amplitude oscillations. No such phenomenon is observed for the upper set of pictures that correspond to the lower amplitude of oscillations.

For $F_{s}=0.234\left(=1.4 F_{o}\right)$, the upstream cylinder still behaves like a single cylinder while the downstream cylinder exhibits oscillations of much lower amplitude, primarily, in the cross-flow direction. The nondimensional frequency associated with the temporal variation of the lift coefficient and cross-flow response of both the cylinders is $0 \cdot 191$. Figure 5 shows the pressure and vorticity fields at a time instant that corressponds to the peak cross-flow location of the upstream cylinder for the fully developed solution. Qualitatively, the flow is very similar to the one in the earlier case. The vortex-shedding frequency for the present 

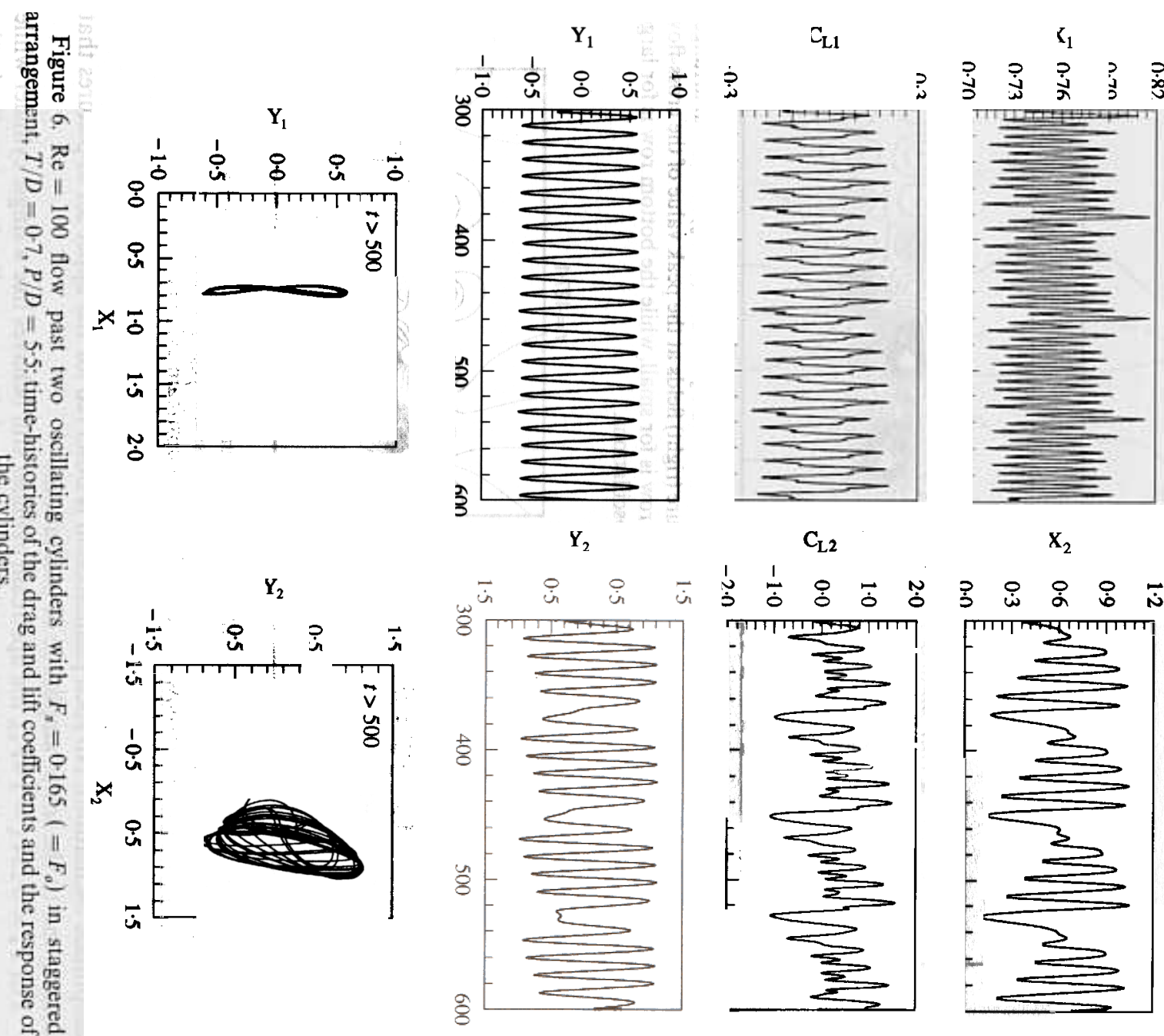

$\mathrm{C}_{\mathrm{DI}}$

$\breve{\infty}$
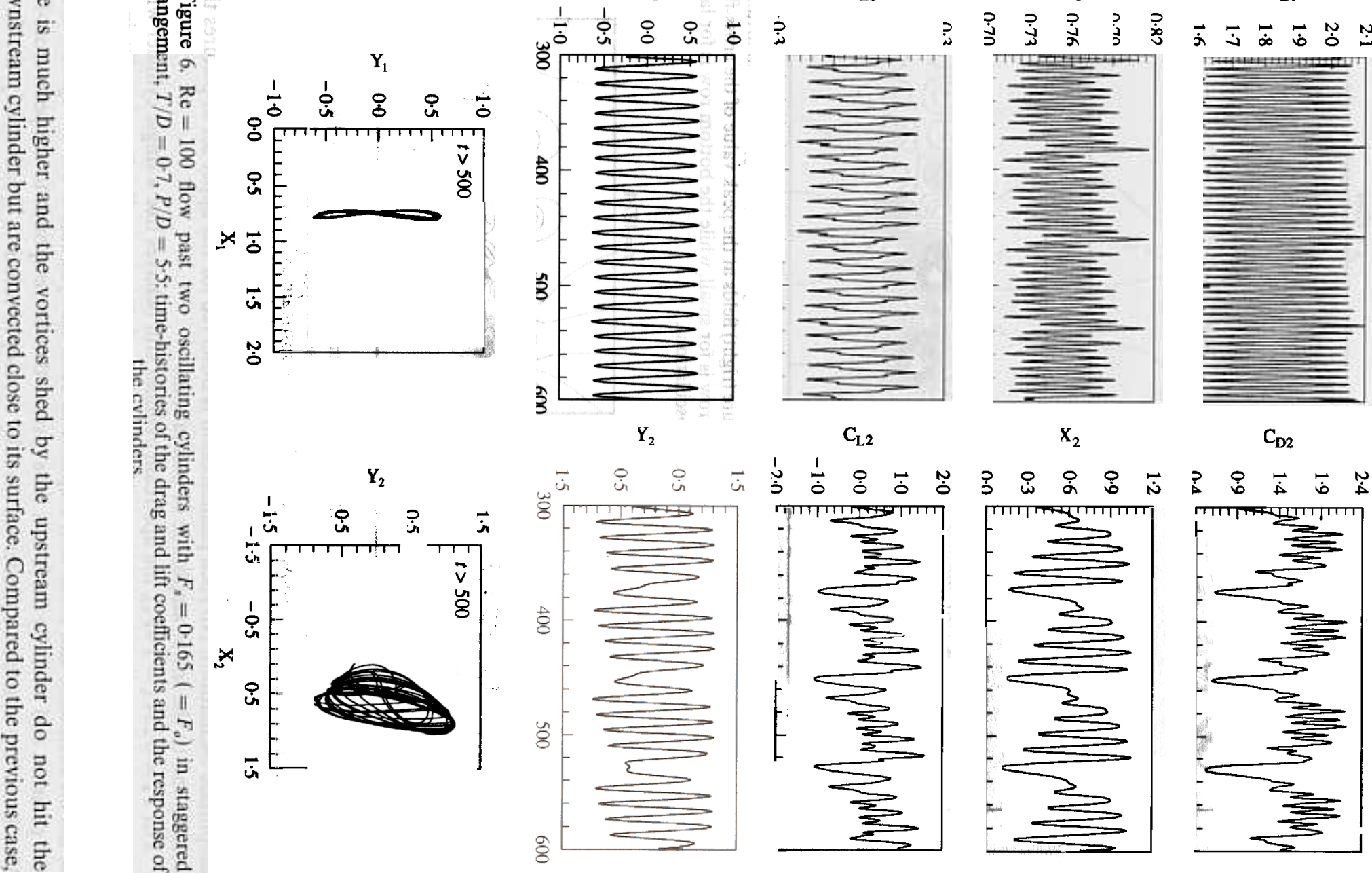

$\mathrm{C}_{\mathrm{D} 2}$
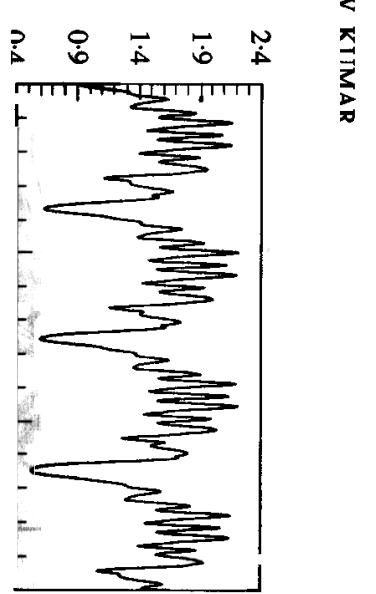

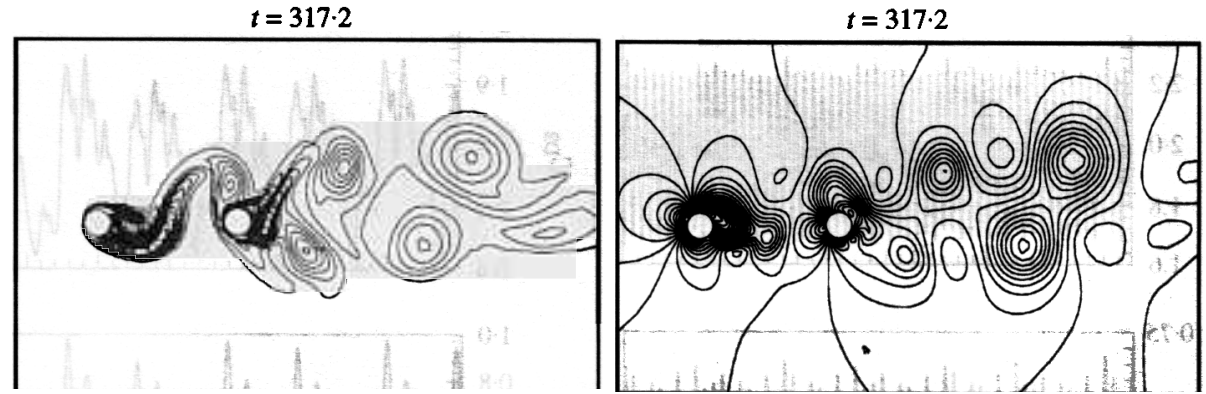

Figure 7. $R e=100$ flow past two oscillating cylinders with $F_{s}=0 \cdot 165\left(=F_{0}\right)$ in staggered arrangement, $T / D=0 \cdot 7, P / D=5 \cdot 5$ : vorticity (left) and pressure (right) fields at the peak value of the cross-flow displacement of the upstream cylinder.

\subsection{Flow Past Two Cylinders in Staggered Arrangement; $T / D=0 \cdot 7, P / D=5 \cdot 5$}

The staggered arrangement of cylinders is the one that is most likely to occur in an engineering situation, for example, in heat exchangers and other cooling systems involving tube bundles. Experimental investigations for such arrangements with stationary cylinders have been reported by various researchers in the past, for example, by Chen (1987), Zdravkovich (1977) and Kiya et al. (1980). Numerical simulations of such flows for $\operatorname{Re}=100$ and 1000 were reported by Mittal et al. (1997) and for past periodic arrays of cylinders at $\operatorname{Re}=100$ by Johnson et al. (1993).

The vortex shedding from the two cylinders in this arrangement is almost synchronized (Mittal et al. 1997). The counterclockwise rotating vortices that are shed from the lower surface of the upstream cylinder pass below the downstream cylinder and interact with the counterclockwise rotating vortex shed from its lower surface. The two coalesce downstream and one can observe a row of counter-clockwise rotating vortices that are elongated along the flow direction. The clockwise rotating vortices that are shed from the upper surface of the upstream cylinder hit the rear one and split into two. The one that glides along the lower surface gets diffused quite soon, while the one that moves towards the upper surface of the downstream cylinder interacts with its clockwise rotating vortex. This interaction produces a stronger vortex that is shed from the upper surface of the second cylinder and a row of such vortices is observed in the wake. The vortex-shedding frequency for both the cylinders in this arrangement is $F_{o}=0 \cdot 165$.

Figure 6 shows the time histories of the lift and drag coefficients and the response of the cylinders for $F_{s}=0.165\left(=F_{o}\right)$ for the fully developed solution. The response of the upstream cylinder is very similar to that of a single cylinder as reported by Mittal \& Kumar (1998). The lift coefficient varies with a nondimensional frequency of 0.155 and the drag oscillates at twice this frequency. Consequently, the trajectory of the cylinder corresponds to a Lissajous figure-of-eight. The behaviour of the downstream cylinder is, however, significantly different. Beats can be observed in the time-histories of the various quantities for the second cylinder. The dominant frequency in the time variation of the lift coefficient and cross-flow vibrations is $0 \cdot 155$. The drag coefficient also varies with the same frequency. Therefore, the trajectory of the cylinder is more like a tilted oval rather than the figure of 8 that is usually observed. The amplitude of the in-line oscillations is much larger than that observed for the tandem arrangement. Soft lock-in is observed for both the cylinders. Figure 7 shows the pressure and vorticity fields at a time instant that corressponds to the peak cross-flow location of the upstream cylinder for the fully developed solution. The 

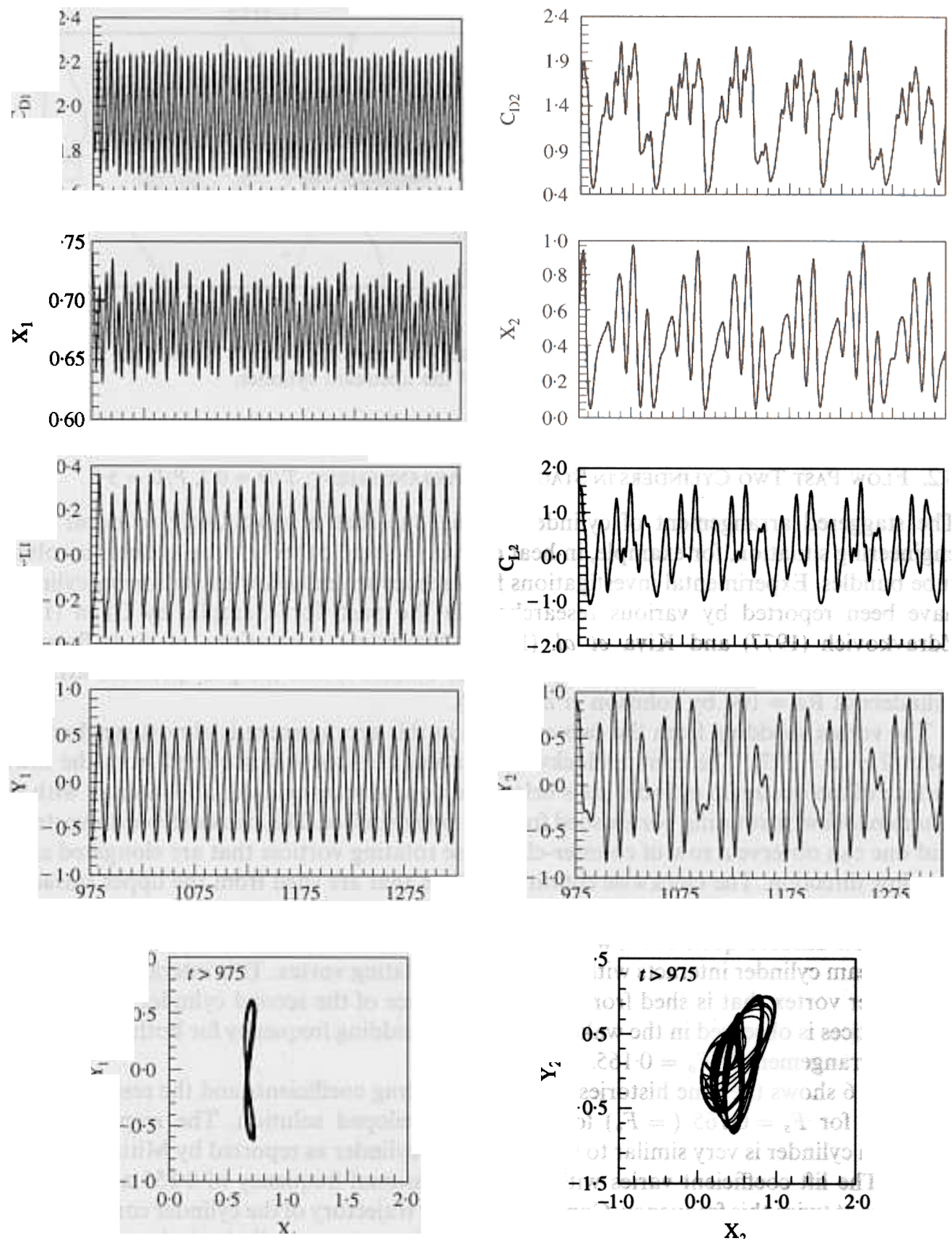

Figure 8. $\operatorname{Re}=100$ flow past two oscillating cylinders with $F_{s}=0 \cdot 18\left(=1.09 F_{o}\right)$ in staggered arrangement, $T / D=0 \cdot 7, P / D=5 \cdot 5$ : time-histories of the drag and lift coefficients and the response of the cylinders.

qualitative nature of the vortex dynamics is similar to that observed for stationary cylinders in this arrangement.

The time histories of the various quantities and the flow pictures for $F_{s}=0.18\left(=1.09 F_{o}\right)$ are shown in Figures 8 and 9, respectively. The behaviour of the system is very similar to 

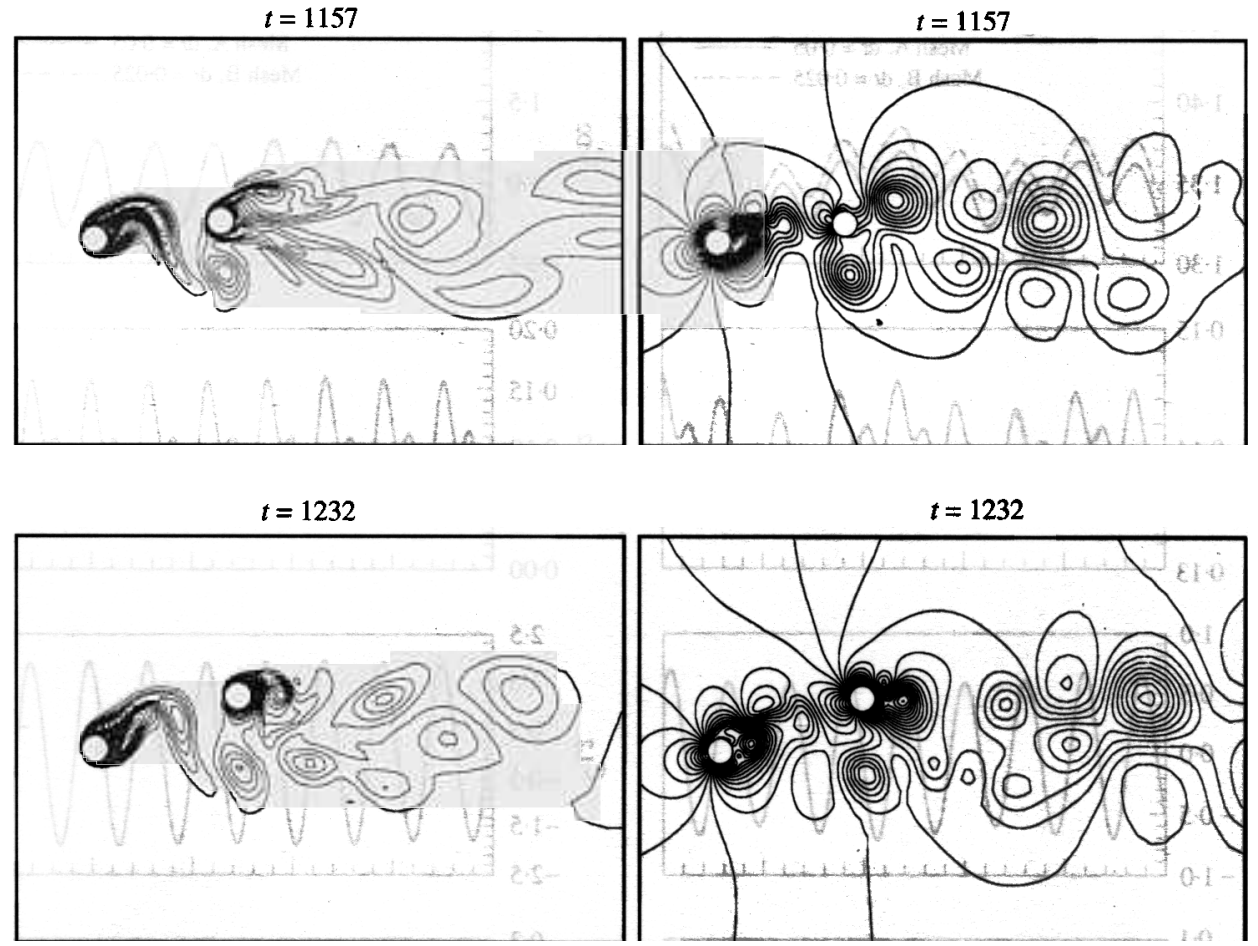

Figure 9. $\operatorname{Re}=100$ flow past two oscillating cylinders with $F_{s}=0.18\left(=1.09 F_{o}\right)$ in staggered arrangement, $T / D=0 \cdot 7, P / D=5 \cdot 5$ : vorticity (left) and pressure (right) fields at the peak value of the cross-flow displacement of the downstream cylinder. The top row is for small, while the bottom row is for large amplitude oscillations.

that observed for $F_{s}=F_{o}$. The flow pictures in Figure 9 correspond to the time instant when the downstream cylinder is at the peak value of the cross-flow displacement. The upper row is for small-amplitude vibrations, while the second row is for large-amplitude oscillations. In both sets of pictures, the vortex shed from the upstream cylinder is positioned below the downstream one and creates a local suction that pulls the cylinder towards it. For the large-amplitude solution this is augmented by a stronger vortex shedding from the second cylinder.

Figure 10 shows the time-histories of the lift and drag coefficients and the response of the cylinders for $F_{s}=0.33\left(=2 F_{o}\right.$ ), for the fully developed solution. The oscillation amplitude of the two cylinders is significantly smaller than those in the previous cases. The upstream cylinder oscillates primarily in the cross-flow direction. The beats, observed in the earlier cases for the response of the downstream cylinder, are not seen in the present solution. However, as before, the dominant frequency in the variation of drag coefficient for the downstream cylinder is the same as that for the lift coefficient. In the case of upstream cylinder, the drag coefficient oscillates with twice the frequency of the variation of the lift coefficient. The trajectory of the upstream cylinder resembles a slender eight while that of the downstream cylinder resembles the shape of a banana.

Also shown in the same figure are the time histories obtained with a more refined finite element mesh and with a smaller time step. The refined mesh consists of 20015 nodes and 19648 elements. It can be observed that the two solutions are quite close. In fact, the time-histories of the displacements of the two cylinders obtained from the 

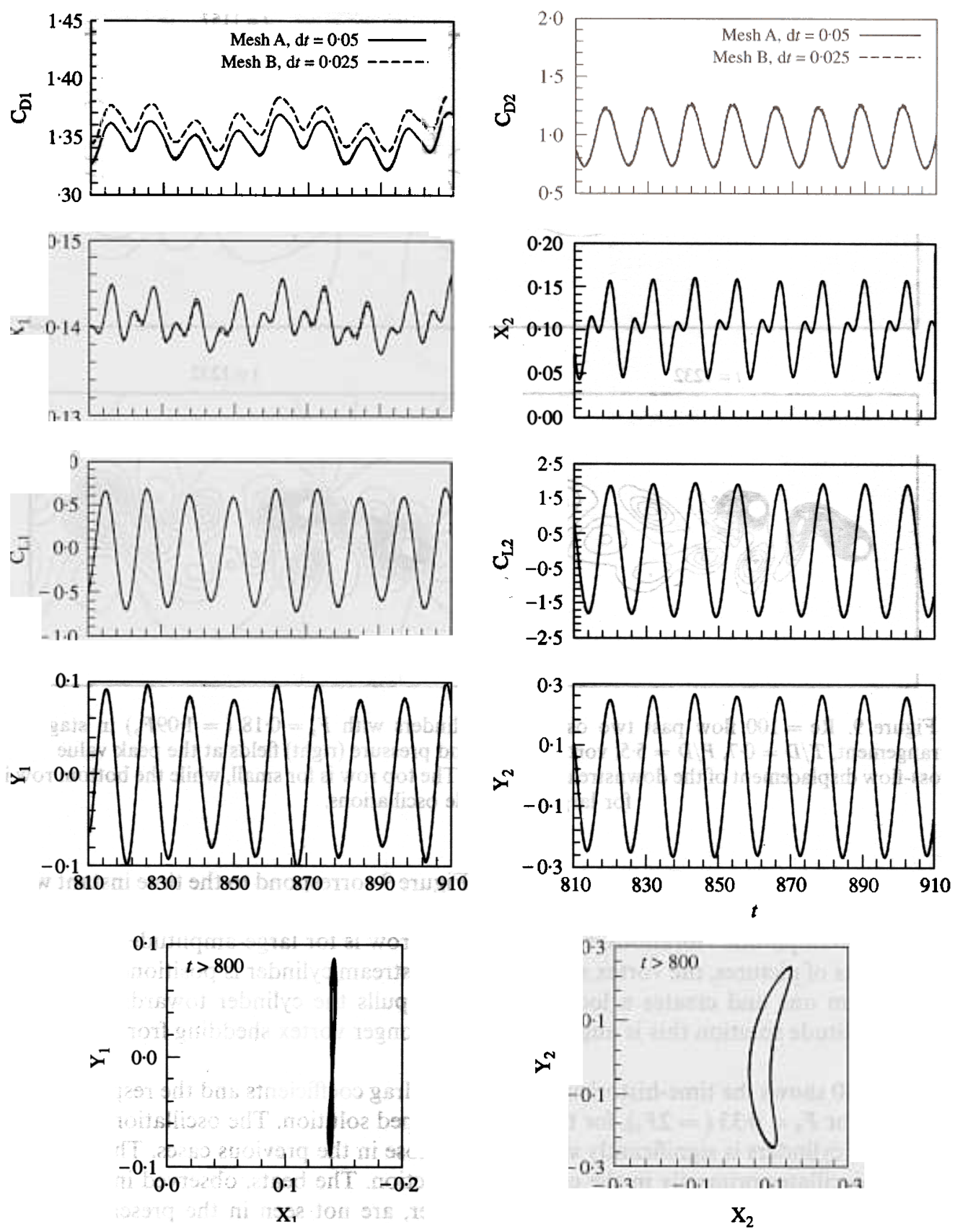

Figure 10. $R e=100$ flow past two oscillating cylinders with $F_{s}=0.33\left(=2 F_{o}\right)$ in staggered arrangement, $T / D=0.7, P / D=5.5$ : time-histories of the drag and lift coefficients and the response of the cylinders (mesh A in solid lines and mesh B in broken lines). Mesh A contains 8552 nodes and 8327 elements while Mesh B consists of 20015 nodes and 19648 elements.

two computations are almost indistinguishable. This demonstrates that the original mesh and time step are adequate to carry out the computations for this flow problem. The spatial as well as temporal convergence of the present results is, therefore, established. Figure 11 shows the pressure and vorticity fields at a time instant that corressponds to the peak 

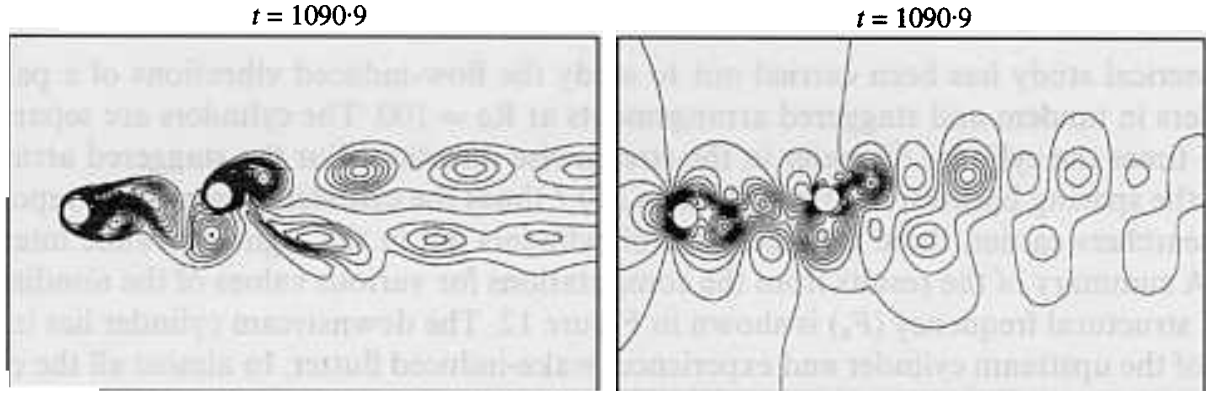

Figure 11. $\operatorname{Re}=100$ flow past two oscillating cylinders with $F_{s}=0.33\left(=2 F_{o}\right)$ in staggered arrangement, $T / D=0 \cdot 7, P / D=5 \cdot 5$ : vorticity (left) and pressure (right) fields at the peak value of the cross-flow displacement of the upstream cylinder.
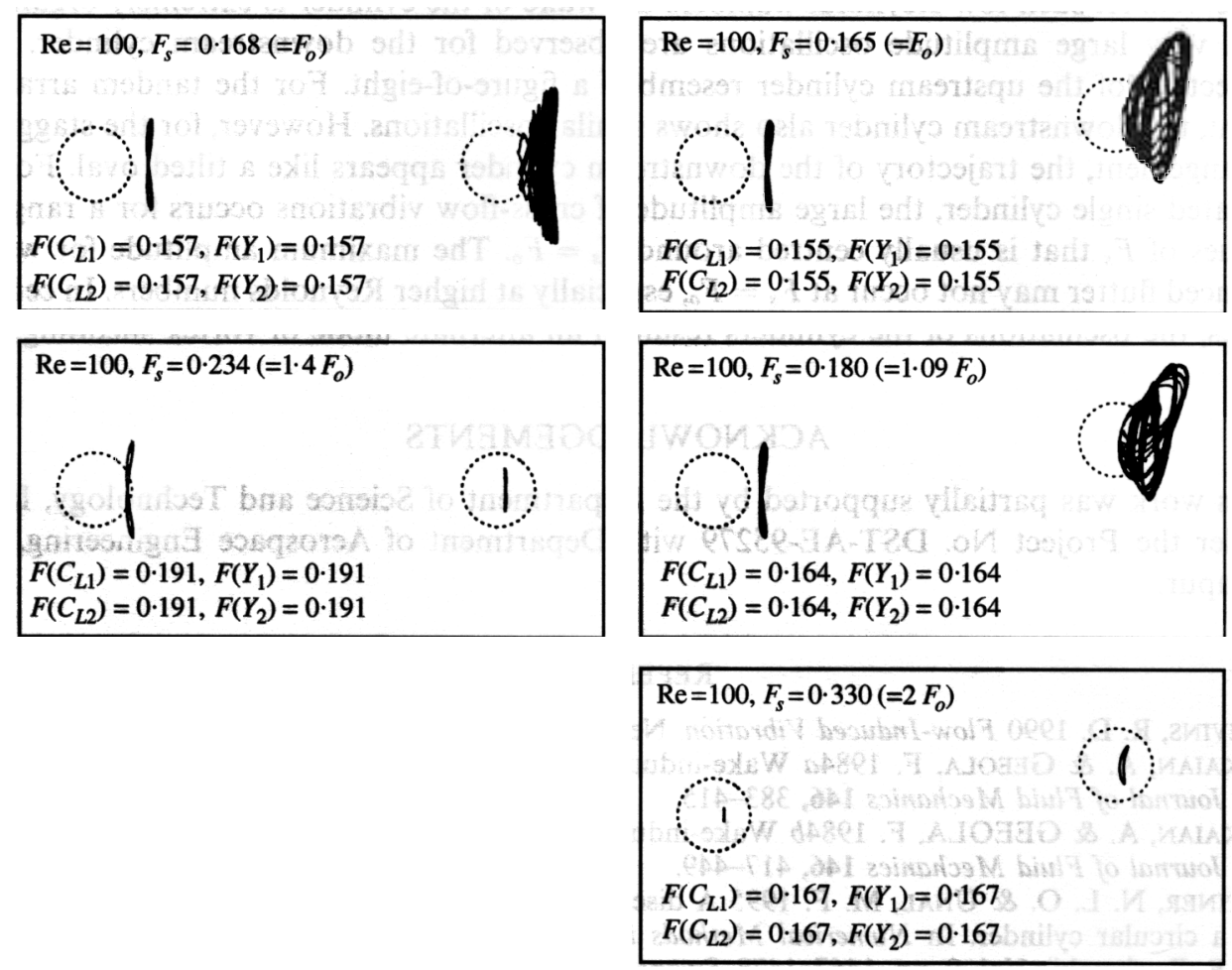

Figure 12. Summary of the flow past two oscillating cylinders. Results for cylinders in tandem $(P / D=5 \cdot 5, T / D=0)$ are on the left and those for the staggered arrangement $(P / D=5 \cdot 5, T / D=0 \cdot 7)$ are on the right. The trajectory of the centre of the cylinders is shown in solid lines while the initial location of the cylinders is shown in dotted lines. Also shown are the dominant frequencies for the time variation of the lift coefficients and the cross-flow oscillations of the two cylinders.

cross-flow location of the upstream cylinder for the fully developed solution. The flow pictures are almost identical to that for stationary cylinders. The vortex-shedding frequency for both the cylinders is 0.167 which is very close to $F_{o}$. 


\section{CONCLUSIONS}

A numerical study has been carried out to study the flow-induced vibrations of a pair of cylinders in tandem and staggered arrangements at $R e=100$. The cylinders are separated by 5.5 times the cylinder diameter in the streamwise direction. For the staggered arrangement, the spacing between the two cylinders is 0.7 times the cylinder diameter. As reported by researchers earlier, these arrangements of cylinders fall in the regime of wake interference. A summary of the results from the computations for various values of the nondimensional structural frequency $\left(F_{s}\right)$ is shown in Figure 12. The downstream cylinder lies in the wake of the upstream cylinder and experiences wake-induced flutter. In almost all the cases the upstream cylinder responds like an isolated single cylinder. Soft lock-in is observed in many cases; the vortex-shedding frequency of the cylinders is detuned from the structural frequency. This is usually observed when the cylinder is immersed in a heavier fluid (like water) and the mass of the oscillator is not too large compared to the mass of the surrounding fluid it displaces. It has been shown earlier that, as the mass of the oscillator is increased, the detuning between the two frequencies reduces and eventually lock-in is observed. At such low Reynolds numbers the wake of the cylinder is extremely organized and very large amplitude oscillations are observed for the downstream cylinder. The trajectory of the upstream cylinder resembles a figure-of-eight. For the tandem arrangement, the downstream cylinder also shows similar oscillations. However, for the staggered arrangement, the trajectory of the downstream cylinder appears like a tilted oval. For an isolated single cylinder, the large amplitude of cross-flow vibrations occurs for a range of values of $F_{s}$ that is usually centred around $F_{s}=F_{o}$. The maximum amplitude for wakeinduced flutter may not occur at $F_{s}=F_{o}$, especially at higher Reynolds numbers. In certain cases, the oscillations of the cylinders result in an alternate mode of vortex shedding.

\section{ACKNOWLEDGEMENTS}

This work was partially supported by the Department of Science and Technology, India under the Project No. DST-AE-95279 with Department of Aerospace Engineering, IIT Kanpur.

\section{REFERENCES}

BLeVINS, R. D. 1990 Flow-Induced Vibration. New York: Van Nostrand Reinhold.

BoKaIAN, A. \& GEeOLA, F. 1984a Wake-induced galloping of two interfering circular cylinders. Journal of Fluid Mechanics 146, 383-415.

BOKAIAN, A. \& GEEOLA, F. $1984 b$ Wake-induced galloping of two interfering circular cylinders. Journal of Fluid Mechanics 146, 417-449.

Cetiner, N. L. O. \& UNAL, M. F. 1995 A discrete vortex study of vortex-induced oscillations of a circular cylinder. In Numerical Methods in Laminar and Turbulent Flow (eds C. Taylor \& P. Durbetaki). Vol. 9, pp. 1467-1478. Swansea. U.K.: Pineridge Press.

ChANG, K. S. \& SA, J. Y. 1992 Patterns of vortex shedding from an oscillating circular cylinder. $A I A A$ Journal 30, 1331-1336.

CHEN, S. S. 1987 Flow-Induced Vibrations of Circular Cylindrical Structures. New York: Hemisphere Publishing Corporation.

Durgin, W. W., MARCh, P. A. \& LeFEBVRe, P. J. 1980 Lower mode response of circular cylinders in cross-flow. ASME Journal of Fluids Engineering, 102, 183-190.

GRIFFIN, O. M. 1971 The unsteady wake of an oscillating cylinder at low Reynolds number. Journal of Applied Mechanics 38, 729-738.

GRIFFIN, O. M. \& RAMBERG, S. E. 1975 Vortex sheddding from a cylinder vibrating in line with an incident uniform flow. Journal of Fluid Mechanics, 75, 257-271.

JendrzejCzYK, J. A., Chen, S. S., \& Wambsganss, M. W. 1979 Dynamic response of a pair of circular tubes subiected to liauid cross flow. Journal of Sound and Vihration 67. 263-273 
Johnson, A. A., TezduYAR, T. E. \& LIOU, J. 1993 Numerical simulation of flows past periodic arrays of cylinders. Computational Mechanics 11, 371-383.

KIM, H. J. \& DURBIN, P. A. 1988 Investigation of the flow between a pair of circular cylinders in the flopping regime. Journal of Fluid Mechanics 196, 431-448.

KING, R. 1977 A review of vortex shedding research and its application. Ocean Engineering 4, 141-171.

KING, R. \& JoHNS, D. J. 1976 Wake interaction experiments with two flexible cirular cylinders in flowing water. Journal of Sound and Vibration 45, 259-283.

KIYA, M., ARIE, M., TAMURA, H., \& MORI, H. 1980 Vortex shedding from two circular cylinders in staggered arrangement. ASME Journal of Fluids Engineering 102, 166-173.

KIYA, M., MOChIZUKI, O., IDO, Y., SUZUKI, T. \& ARAI, T. 1992 Flip-fiopping flow around two bluff bodies in tandem arrangement. In Bluff-Body Wakes, Dynamics and Instabilities (eds $\mathrm{H}$. Eckelmann, J. M. R. Graham, P. Huerre \& P. A. Monkewitz) IUTAM Symposium, pp. 15-18, Göttingen: Springer-Verlag.

KNISELY, C. W. \& KAWAGOE, M. 1990 Force-displacement measurements on closely spaced tandem cylinders. In Bluff Body Aerodynamics and its Applications (eds M. Ito, M. Matsumoto \& N. Shiraishi) pp. 81-90. Amsterdam: Elsevier.

KOOPMANN, G. H. 1967 The vortex wakes of vibrating cylinders at low Reynolds numbers. Journal of Fluid Mechanics 28, 501-512.

Lecointe, Y., PiQuet, J. \& Plantec, J. 1987 Flow structure in the wake of an oscillating cylinder. In Forum on Unsteady Flow Separation (ed. K. N. Ghia) FED-52, pp. 147-157. New York: ASME.

Matsumoto, M., Shiraishi, N. \& ShIRATo, H. 1990 Aerodynamic instabilities of twin circular cylinders. In Bluff Body Aerodynamics and its Applications (eds M. Ito, M. Matsumoto \& N. Shiraishi) pp. 91-100. Amsterdam: Elsevier.

MITTAL, S. 1992 Stabilized space-time finite element formulations for unsteady incompressible flows involving fluid-body interactions Ph.D. Thesis, University of Minnesota, U.S.A.

MITTAL, S. \& KumAR, V. 1998 Flow induced vibrations of a light circular cylinder at Reynolds numbers $10^{3}$ to $10^{4}$. Journal of Sound and Vibration, accepted.

MitTAL, S. \& KuMAR, V. 1999 Finite element study of vortex-induced cross-flow and in-line oscillations of a circular cylinder at low Reynolds numbers. International Journal for Numerical Methods in Fluids 31, 1087-1120.

MITTAL, S., KUMAR, V. \& RAGHUVANSHI, A. 1997 Unsteady incompressible flow past two cylinders in tandem and staggered arrangements. International Journal for Numerical Methods in Fluids 25, 1315-1344.

Mittal, S., Ratner, A., Hastreiter, D. \& Tezduyar, T. E. 1991 Space-time finite element computation of incompressible flows with emphasis on flows involving oscillating cylinders. International Video Journal of Engineering Research 1, 83-96.

MitTAL, S. \& TezduYAR, T. E. 1992 A finite element study of incompressible flows past oscillating cylinders and airfoils. International Journal for Numerical Methods in Fluids 15, 1073-1118.

MITTAL, S. \& TEZDUYAR, T. E. 1994 Massively parallel finite element computation of incompressible flows involving fluid-body interactions. Computer Methods in Applied Mechanics and Engineering $112,253-282$.

Mittal, S. \& Tezduyar, T. E. 1995 Parallel finite element simulation of 3D incompressible flows: fluid-structure interactions. International Journal for Numerical Methods in Fluids 21, 933-953.

OLINGER, D. J. \& SREENIVASAN, K. R. 1988 Nonlinear dynamics of the wake of an oscillating cylinder. Physical Review Letters 60, 797-800.

ONGoren, A. \& RoCKwell, D. 1988 a Flow structure from an oscillating cylinder. Part 1: Mechanisms of phase shift and recovery in the near wake. Journal of Fluid Mechanics 191, 197-223.

ONGOREN, A. \& ROCKWELL, D. $1988 b$ Flow structure from an oscillating cylinder. Part 2: Mode competition in the near wake. Journal of Fluid Mechanics 191, 225-245.

SAAD, Y. \& SCHULTZ, M. 1986 GMRES: A generalized minimal residual algorithm for solving nonsymmetric linear systems. SIAM Journal of Scientific and Statistical Computing 7, 856-869.

SARPKAYA, T. 1979 Vortex-induced oscillations: a selective review. Journal of Applied Mechanics 46, 241-258.

TANidA, Y., OKaJima, A. \& WATANABE, Y. 1973. Stability of a circular cylinder in uniform flow or in a wake. Journal of Fluid Mechanics 61, 769-784.

TeZDUYAR, T. E., BEHR, M. \& LIOU, J. 1992a A new strategy for finite element computations involving moving boundaries and interfaces - the deforming-spatial-domain/space-time procedure: I. The concept and the preliminary tests. Computer Methods in Applied Mechanics and Engineering 94, 339-351. 
Tezduyar, T. E., BeHR, M., MitTal, S. \& LiOU, J. $1992 b$ A new strategy for finite element computations involving moving boundaries and interfaces-the deforming-spatialdomain/space-time procedure: II. Computation of free-surface flows, two-liquid flows, and flows with drifting cylinders. Computer Methods in Applied Mechanics and Engineering 94, 353-371.

TezduYar, T. E., MitTal, S., RaY, S. E. \& SHIH, R. 1992 Incompressible flow computations with stabilized bilinear and linear equal-order-interpolation velocity-pressure elements. Computer Methods in Applied Mechanics and Engineering 95, 221-242.

TOEBES, G. H. 1969 The unsteady flow and wake near an oscillating cylinder. ASME Journal of Basic Engineering 91, 493-505.

TokunaGa, H., TANAKA, T. \& SATOFUKA, N. 1992 Numerical simulation of viscous flows along multiple bodies by generalized vorticity-stream function formulation. Computational Fluid Dynamics Journal 1, 58-66.

Tsui, Y. T. 1977 On wake-indiced flutter of a circular cylinder in the wake of another. Journal of Applied Mechanics, Transactions of the ASME 99, 194-200.

WilliAMSON, C. H. K. 1985 Evolution of a single wake behind a pair of bluff bodies. Journal of Fluid Mechanics 159, 1-18.

WILliamson, C. H. K. 1996 Vortex dynamics in the cylinder wake. Annual Review of Fluid Mechanics 28, 477-539.

Williamson, C. H. K. \& Roshko, A. 1988 Vortex formation in the wake of an oscillating cylinder. Journal of Fluids and Structures 2, 355-381.

WU, J. C. \& HU, Y. C. 1993 Flow characteristics of tandem circular cylinders: Effects of diameter ratio and longitudinal spacing. AIAA Paper 93-3088, AIAA 24th Fluid Dynamics Conference, Orlando, FL, U.S.A.

ZDRAVKOVICH, M. M. 1977 Review of flow interference between two circular cylinders in various arrangements. ASME Journal of Fluids Engineering 99, 618-633.

ZDRAVKOVICH, M. M. 1985 Flow induced oscillations of two interfering circular cylinders. Journal of Sound and Vibration 101, 511-521. 